\title{
Mise en évidence de souches de Trichoderma spp à la fois antagonistes de Pythium ultimum et stimulatrices de la croissance des plantes
}

\author{
O Besnard 1, P Davet 2* \\ 1 Société de nouvelle fertilisation Letellier, BP 1233, F 34011 Montpellier Cedex 1; \\ 2 Laboratoire de biologie et pathologie végétale, INRA-ENSA, F34060 Montpellier Cedex i, France
}

(Reçu le 8 février 1993; accepté le 15 février 1993)

\begin{abstract}
Résumé - Un tri réalisé parmi 113 isolats de Trichoderma spp appartenant à divers groupes d'espèces (sensu Rifai) a permis de trouver 7 souches dont les filtrats de culture sont capables d'augmenter la vitesse et le taux de germination de tomates et de concombres in vitro et 4 souches manifestant une forte activité antagoniste vis-à-vis de Pythium ultimum. Deux souches au moins, AGI (T polysporum) et BR 105 ( $T$ harzianum résistant au bénomyl) possèdent les deux types d'activité. Ces résultats sont confirmés par des essais en serre dans des pots contenant un mélange autoclavé de tourbe et de vermiculite. Dans ces conditions BR 105, ajouté à raison de $10^{7}$ propagules $\mathrm{g}^{-1}$, améliore la levée $(+31$ et $+25 \%)$, la masse $(+72$ et $+68 \%)$ et la production de fleurs à l'âge de 2 mois $(+84$ et $+236 \%$ ) de tomates et de concombres, respectivement. Dans un substrat contaminé par $P$ ultimum, l'incorporation de BR 105 augmente le taux de levée des concombres de $138 \%$ et le nombre de fleurs à 2 mois de $183 \%$. Enfin, la quantité de pensées fleuries, $47 \mathrm{j}$ après leur repiquage chez un horticulteur dans un substrat organique du commerce, est plus élevée en présence de BR $105(+83 \%)$. Cependant, un traitement fongicide du terreau permet d'obtenir un résultat du même ordre. L'interprétation de résultats de cette sorte doit donc être faite avec prudence.
\end{abstract}

Trichoderma / Pythium ultimum / antagonisme microbien / stimulation

Summary - Observations on some Trichoderma spp strains that are simultaneously antagonistic to Pythium ultimum and growth stimulating. One hundred and thirteen Trichoderma spp isolates belonging to several species aggregates (sensu Rifai) were screened. Culture filtrates from 7 of these isolates were found capable of increasing germination speed and rate of tomato and cucumber seeds in vitro; 4 isolates exhibited high antagonistic activity against Pythium ultimum in Petri dishes. At least 2 strains, AGI (T polysporum) and BR 105 (a benomyl-resistant Tharzianum) showed both types of biological activity. These results were confirmed in greenhouse trials. When plants were seeded in pots filled with an autoclaved mixture of peat and vermiculite, addition of BR $105\left(10^{7} \mathrm{cfu}^{-1}\right)$ improved emergence rate $(+31$ and $+25 \%)$, dry mass $(+72$ and $+68 \%)$ and flower setting at 2 months $(+84$ and $+236 \%)$ of tomatoes and cucumbers, respectively. In a $\mathrm{P}$ ultimum-infested substrate, emergence rates of cucumber and flower production by 2-month-old plants were increased by 138 and 183\% respectively in pots treated with BR 105 . Finally, pansies grown in a potting mixture in a commercial greenhouse had more flowering plants (+83\%), $47 d$ after planting out when BR 105 had been incorporated into the substrate. However, a similar result was obtained through substrate treatment with a fungicide. Consequently, the interpretation of this kind of results should be made with care.

Trichoderma / Pythium ultimum / microbial antagonist / stimulation

\section{INTRODUCTION}

Dans les cultures sous abri, la forte densité de plantation, les conditions de production intensive et l'utilisation de variétés à hautes performances, de plus en plus éloignées des types rustiques, créent des conditions favorables au développement des maladies. L'abandon des cultures en pleine terre et l'adoption de substrats artificiels ont permis de limiter l'incidence des maladies dues à des champignons du sol. Mais l'humidité permanente entretenue au pied

\footnotetext{
* Correspondance et tirés à part
} 
des plantes par les systèmes d'arrosage tend à favoriser certaines catégories de parasites qui, malgré les précautions prises, demeurent un risque constant. C'est ainsi que les Pythium spp, responsables de fontes de semis et d'accidents de levée, demeurent une des préoccupations majeures des maraîchers et des horticulteurs.

L'efficacité de la protection chimique n'est pas toujours satisfaisante, et ses effets sur l'environnement ne sont pas négligeables. Cependant la possibilité d'une protection par des auxiliaires biologiques devient une solution alternative envisageable. Durant ces dernières années, un grand nombre de bactéries et de champignons antagonistes des Pythium spp ont ainsi été mis en évidence (Whipps et Lumsden, 1991) mais les résultats les plus intéressants et les plus solidement établis ont été obtenus avec des Trichoderma spp (Sivan et al, 1984; Lifshitz et al, 1986; Harman et al, 1989).

II se trouve par ailleurs que certaines souches de Trichoderma spp semblent exercer une action stimulatrice sur la croissance des plantes, aussi bien in vitro, dans des conditions contrôlées et en l'absence de tout agent pathogène, que dans des substrats de culture (Baker, 1988). Curieusement, ces résultats intéressants n'ont pas donné lieu à d'autres recherches, hormis les travaux tout récemment publiés de Lynch et al (1991) et de Kleifeld et Chet (1992).

II est certain qu'il serait extrêmement avantageux de pouvoir disposer d'un clone de Trichoderma qui soit à la fois protecteur et stimulateur de croissance. Les résultats que nous nous proposons d'exposer montrent qu'il est effectivement possible de trouver de telles souches.

\section{MATÉRIEL ET MÉTHODES}

\section{Origine des champignons utilisés}

Une collection de 100 souches de Trichoderma, isolées à partir de sols de différentes régions de France, a été utilisée dans les tests préliminaires. La majorité appartiennent au groupe d'espèces $T$ harzianum Rifai. Quelques isolats appartiennent aux groupes $T$ hamatum (Bon) Bain, $T$ aureoviride Rifai, $T$ viride Pers ex S F Gray et $T$ polysporum (Link ex Pers) Rifai. Ces souches proviennent des collections des laboratoires de pathologie végétale de I'INRA de Bordeaux et de Montpellier. Par ailleurs, une suspension conidienne d'une culture monospore (B 11) obtenue à partir d'un $T$ harzianum du sol a été exposée pendant 2 min à un rayonnement ultra-violet de 200 $\mu \mathrm{W} \mathrm{cm} \mathrm{cm}^{-2}$ (Billette, travaux non publiés). Certaines des conidies irradiées ont produit des mutants résistants au bénomyl, dont 13 ont été utilisés dans nos essais comparatifs.

Pour toutes les études du pouvoir antagoniste des souches de Trichoderma nous avons utilisé une souche de Pythium ultimum Trow var ultimum, isolée sur racines de soja dans la région de Toulouse.

\section{Sélection des souches in vitro}

\section{Stimulation de croissance}

Les souches de Trichoderma sont cultivées en erlenmeyer dans le milieu $\mathrm{S}$ liquide de Messiaen et Lafon (1965) additionné de $1 \mathrm{~g} \mathrm{I}^{-1}$ de $\mathrm{Ca} \mathrm{Cl}_{2}$ (Gindrat, 1977). Les liquides de culture sont récoltés après $10 \mathrm{j}$ d'incubation sur un plateau oscillant à la température du laboratoire. Après filtration sur mousseline, les liquides sont stérilisés par passage sur un filtre stérile à pores de $0,45 \mu \mathrm{m}$ de diamètre. Cinq millilitres de filtrat sont répartis à la surface d'une boîte de Petri contenant de l'eau gélosée solidifiée. Les témoins reçoivent $5 \mathrm{ml}$ de milieu $S$ non ensemencé.

Sur chaque boîte sont déposées 10 graines de tomate (cv Saint Pierre) ou de concombre (cv Le Généreux). Les graines sont préalablement désinfectées par trempage pendant 1 min dans une solution d'hypochlorite de sodium à $0,5 \%$, puis rincées 2 fois à l'eau distillée stérile et séchées. Les boîtes sont mises en incubation à l'obscurité à $24^{\circ} \mathrm{C}$. Chaque essai comprend 4 répétitions. Les essais de confirmation, portant sur un nombre plus restreint de souches, comprennent 8 répétitions.

Après 1 semaine d'incubation, on mesure le pourcentage de germination et la masse des plantes après séchage pendant $3 \mathrm{j}$ à l'étuve à $50^{\circ} \mathrm{C}$.

\section{Activité antagoniste}

La méthode est comparable à celle utilisée par Lynch (1987). Un implant de Trichoderma et un implant de $P$ ultimum sont déposés à la surface de boîtes de Petri de $9 \mathrm{~cm}$ de diamètre contenant $10 \mathrm{ml}$ de milieu PDA. Les implants sont placés à $5 \mathrm{~cm}$ l'un de l'autre, selon une ligne diamétrale. Les boîtes, comprenant 4 répétitions par souche, sont incubées à l'obscurité à $20^{\circ} \mathrm{C}$. Trente-six heures après l'ensemencement, on note la présence de zones d'inhibition ou l'interpénétration des cultures ; le développement des 2 champignons est apprécié en planimétrant la surface occupée par leurs thalles respectifs, au moyen d'une caméra couplée à un ordinateur. Après un premier tri, 4 des meilleurs clones et 5 des moins bons choisis au hasard sont confrontés à $P$ ultimum et à 2 autres champignons parasites : Rhizoctonia solani Kühn et Sclerotium rolfsii Sacc. 


\section{Essais en serre}

\section{Conditions générales}

Les souches de Trichoderma sélectionnées à la suite des essais in vitro sont multipliées en fioles de Roux (pour une fiole : $200 \mathrm{~g}$ de sable lavé, $6 \mathrm{~g}$ de farine d'avoine, $30 \mathrm{ml}$ d'eau distillée; 2 autoclavages à $120^{\circ} \mathrm{C}$ pendant $30 \mathrm{~min}$ à $48 \mathrm{~h}$ d'intervalle). Les fioles sont incubées pendant 15 j à $24^{\circ} \mathrm{C}$ à l'obscurité.

L'inoculum est incorporé dans un mélange à parties égales de tourbe blonde fine et de vermiculite stérilisé par 2 autoclavages à $120^{\circ} \mathrm{C}$ pendant $30 \mathrm{~min}$. La quantité d'inoculum est calculée de façon à obtenir $10^{7}$ propagules par $g$ de substrat. Les mélanges sont répartis dans des pots de $15 \times 15 \times 15 \mathrm{~cm}$. Dans chaque pot sont semées 10 graines de tomate (cv Saint Pierre) ou de concombre (cv Le Généreux). Les graines sont préalablement désinfectées superficiellement par trempage dans l'hypochlorite de sodium à $0,5 \%$ pendant $1 \mathrm{~min}$, puis dans l'acide peracétique à $2 \%$ pendant $20 \mathrm{~min}$. Elles sont ensuite rincées 2 fois dans de l'eau stérile, puis égouttées. Les pots sont arrosés à l'eau distillée pendant les 10 premiers jours après le semis. Ils sont ensuite arrosés avec une solution fertilisante, d'abord tous les 2 jours, puis tous les jours. Les pots sont disposés dans une serre, en blocs randomisés.

\section{Étude de l'activité stimulatrice de croissance}

Le mélange du substrat avec les auxiliaires est réalisé 2 semaines avant le semis. Les clones actifs sont comparés à deux témoins constitués, l'un par du substrat non ensemencé, l'autre par un mélange de substrat avec une souche choisie au hasard parmi celles qui n'ont pas manifesté d'effet stimulant dans les essais in vitro.

Les pots sont maintenus à la température de 26 $\pm 2{ }^{\circ} \mathrm{C}$ pendant toute la durée de l'essai. Les taux de levée sont calculés lorsque les plantes ont 2 vraies feuilles (soit 21 j après le semis). Le nombre de plantes est alors ramené à 2 par pot. Les individus excédentaires, arrachés avec précaution, sont rincés pour les débarrasser du substrat adhérent, puis leur masse est déterminée après séchage à l'étuve à $50^{\circ} \mathrm{C}$ pendant $3 \mathrm{j}$. À la fin de l'essai, c'est-à-dire environ 2 mois après le semis, les fleurs sont comptées, et les plantes restantes sont pesées comme précédemment.

Un essai est aussi mis en place chez un horticulteur. Des pensées (Viola tricolor cv Diva), achetées en godets au stade 5 feuilles chez un grossiste, sont repiquées dans des pots de $9 \times 9 \times 8 \mathrm{~cm}$ contenant un substrat commercial à base de tourbe et de sphaignes, à $\mathrm{pH}=6$. Le substrat a préalablement subi l'un des 3 traitements suivants : incorporation de la souche BR 105 à raison de $10^{10}$ propagules par pot (soit environ $15 \times 10^{6}$ propagules $\mathrm{ml}^{-1}$ de substrat); traitement à l'oxyquinoléine à la dose préconisée de $2100 \mathrm{~g} \mathrm{ha}^{-1}$ (soit 1,7 mg par pot); incorporation de la BR 105 et traitement à l'oxyquinoléine. Un quatrième lot, non traité, sert de référence. Les plantes, réparties en 4 blocs comprenant chacun 16 plantes par traitement, sont placées dans une serre parmi les autres pensées. Les relevés commencent 1 mois plus tard à raison d'un, puis 2, par semaine et sont poursuivis jusqu'au stade de la commercialisation, qui a lieu $47 \mathrm{j}$ après le repiquage, juste au début de la floraison. On compte le nombre de plantes subsistantes et le nombre de fleurs formées.

\section{Étude de l'activité antagoniste}

Le mélange du substrat avec les souches de Trichoderma est réalisé, comme précédemment, 2 semaines avant le semis. Une semaine avant le semis, ces mélanges sont infectés avec une souche de $P$ ultimum. Les témoins sont constitués par du terreau non ensemencé et par du terreau contenant $P$ ultimum seul.

L'inoculum de $P$ ultimum est préparé sur farine de maîs gélosée (farine de maïs : $17 \mathrm{~g}$, gélose : $20 \mathrm{~g}$, eau distillée : 1 I) en boîtes de Petri. Après une semaine d'incubation à $20^{\circ} \mathrm{C}$, les cultures sont broyées dans une petite quantité d'eau distillée stérile. Le pathogène est introduit dans le substrat à la dose de 250 propagules (sporanges ou oosphères) par g. La température de la serre est maintenue à $20 \pm 2{ }^{\circ} \mathrm{C}$ pendant la durée de l'essai. II y a 16 pots pour chaque traitement.

Le nombre de plantes dans chaque pot est déterminé 21 j après le semis. Ce nombre est alors ramené à 2 par pot. Les individus excédentaires sont arrachés et lavés, puis mesurés et pesés comme dans les essais précédents. Au bout de 8 semaines, on compte le nombre de fleurs et les plantes sont mesurées et pesées.

\section{Évolution des populations dans les substrats}

Le milieu sélectif et la méthode de Ricci et al (1976) sont utilisés pour l'estimation des populations de $P$ ultimum. Ce milieu contient du bénomyl qui inhibe les populations "sauvages" de Trichoderma. Pour les échantillons dans lesquels la souche résistante BR 105 est présente, on remplace le bénomyl par du triadiménol (15 $\left.\mathrm{mg}^{-1}\right)$ qui inhibe son développement sans empêcher celui de $P$ ultimum. Les populations de Trichoderma sont estimées à l'aide du milieu sélectif et de la méthode de Davet (1979). Pour chaque mesure, l'échantillon moyen est constitué du mélange de prises d'environ $200 \mathrm{ml}$ de substrat, provenant de 4 pots différents d'un même traitement.

Les populations de Trichoderma et de $P$ ultimum sont ainsi évaluées à la fin de l'essai concernant l'activité antagoniste. Pour avoir une idée plus précise de l'évolution de ces populations, un deuxième essai est réalisé, dans lequel les champignons, seuls ou en combinaison, sont incorporés au substrat et répartis dans des pots où aucune graine n'est semée. Dans 
cet essai, l'inoculum de $P$ ultimum (250 prop $\mathrm{g}^{-1}$ ) est introduit dans le terreau $10 i$ après l'inoculum de Trichoderma $\left(10^{8}\right.$ prop $\left.\mathrm{g}^{-1}\right)$. Les pots sont disposés dans une serre à $20 \pm 2{ }^{\circ} \mathrm{C}$ et arrosés de façon à obtenir une teneur en eau correspondant à $70 \%$ de la capacité de rétention. Les populations sont estimées 0,15 et $48 \mathrm{j}$ après l'incorporation de $P$ ultimum, chaque fois dans 2 pots.

En outre, $200 \mathrm{ml}$ de substrat sont prélevés après 0 , 5 , 15 et $84 \mathrm{j}$, chaque fois dans 2 pots préparés comme précédemment. Après séchage à l'air, ces échantillons sont broyés et tamisés à la maille de $1 \mathrm{~mm}$, puis additionnés de poudre de flocons d'avoine $\left(20 \mathrm{~g}^{-1}\right)$. Chaque mélange est alors déposé autour du collet d'une douzaine de jeunes plants de concombre âgés de $6 \mathrm{j}$, préalablement semés dans du terreau stérilisé ajusté à $80 \%$ de la capacité de rétention en eau (Bouhot et Ellal, 1976). Les concombres sont maintenus $24 \mathrm{~h}$ à $15^{\circ} \mathrm{C}$ puis placés dans une pièce à $20^{\circ} \mathrm{C}$. Le taux de mortalité des concombres est déterminé $6 \mathrm{j}$ après l'apport de l'amendement.

\section{RÉSULTATS}

\section{Stimulation de croissance}

\section{Essais in vitro}

L'étude préliminaire portant sur l'ensemble de la collection de Trichoderma montre que presque tous les filtrats de culture $(92,9 \%$ des isolats $)$ sont sans effet, ou exercent même un effet dépressif sur la germination des graines de tomate et de concombre (résultats non présentés). Pour quelques isolats cependant, les taux de germination et la production de matière sèche sont supérieurs à ceux des témoins.

Nous avons alors comparé les 7 meilleures et les 4 moins bonnes souches mises en évidence à l'issue de ce premier tri. Les résultats (tableau I) confirment la validité du premier classement. Deux souches de $T$ polysporum (AGI et $\mathrm{Jl} 1$ ) et un des clones résistant au bénomyl issus de $B$ 11 (BR 105) paraissent particulièrement intéressants. À l'opposé, la souche ZK se montre nettement inhibitrice.

\section{Essai en serre}

La souche $\mathrm{Jl} 1$ ayant un développement lent et une faible sporulation, seules AGl et BR 105 ont été retenues. Nous les avons comparées à la souche Tsp2, choisie au hasard parmi les 4 moins bonnes.

On constate (tableau II) que les souches AGI et BR 105 améliorent significativement la levée des tomates et des concombres, le gain relatif par rapport au témoin pouvant atteindre $30 \%$. Dans les pots contenant AGI ou BR 105, la masse des plantes âgées de 3 semaines, mesurée après séchage, est supérieure de 50 à 100\%

Tableau I. Effet in vitro des filtrats de culture de 11 isolats de Trichoderma sur le taux de germination (après 1 semaine d'incubation à $24^{\circ} \mathrm{C}$ ) et sur la production de matière sèche de tomates et de concombres (moyennes de 8 répétitions). Dans chaque colonne, les pourcentages suivis d'une même lettre ne sont pas différents au seuil de $5 \%$, selon le test de Newman et Keuls.

\begin{tabular}{|c|c|c|c|c|}
\hline \multirow[t]{2}{*}{ Souches } & \multicolumn{2}{|c|}{ Pourcentages de germination } & \multicolumn{2}{|c|}{ Matière sèche (mg) } \\
\hline & Tomate & Concombre & Tomate & Concombre \\
\hline BR 105 & $97,5 \mathrm{a}$ & 86,3 a & $125,7 \mathrm{a}$ & $780,2 \mathrm{a}$ \\
\hline AGI & $83,8 \mathrm{~b}$ & $71,3 \mathrm{~b}$ & $120,4 \mathrm{ab}$ & $772,7 \mathrm{ab}$ \\
\hline Jl 1 & $87,5 b$ & $80,0 \mathrm{ab}$ & $127,4 \mathrm{a}$ & 787,6 a \\
\hline ABT & $78,8 \mathrm{bc}$ & $72,5 b$ & $125,1 \mathrm{a}$ & $765,3 a b$ \\
\hline LR & $77,5 \mathrm{bc}$ & 86,3 a & $121,5 \mathrm{a}$ & $765,0 \mathrm{ab}$ \\
\hline$R L$ & $85,0 \mathrm{~b}$ & $73,8 \mathrm{~b}$ & & $750,0 \mathrm{~b}$ \\
\hline QJ & $80,3 \mathrm{~b}$ & $71,8 \mathrm{~b}$ & & $750,0 \mathrm{~b}$ \\
\hline HH 15 & $75,8 \mathrm{bc}$ & & $115,6 b c$ & $743,0 \mathrm{~b}$ \\
\hline QN & $73,5 \mathrm{bc}$ & $68,8 \mathrm{~b}$ & $118,0 \mathrm{~b}$ & $750,0 \mathrm{~b}$ \\
\hline Tsp 2 & $66,8 \mathrm{~cd}$ & $67,5 b$ & $115,3 \mathrm{bc}$ & $740,0 \mathrm{~b}$ \\
\hline $\mathrm{ZK}$ & $60,0 \mathrm{~d}$ & $67,5 b$ & $96,8 \mathrm{~d}$ & $728,0 \mathrm{c}$ \\
\hline Témoin & $75,0 \mathrm{bc}$ & $68,8 \mathrm{~b}$ & $118,0 \mathrm{~b}$ & $743,0 \mathrm{~b}$ \\
\hline
\end{tabular}


Tableau II. Effet en pots à la serre de l'incorporation de souches de Trichoderma dans le substrat de culture sur les taux de germination de tomates et de concombres (moyennes de 16 répétitions). Dans chaque colonne, les pourcentages suivis d'une même lettre ne sont pas différents au seuil de $5 \%$, selon le test de Newman et Keuls. Les chiffres indiqués entre parenthèses représentent les pourcentages de variation par rapport au témoin.

Traitements $\begin{array}{ll}\text { Tomate } \\ (1990)\end{array} \quad \begin{aligned} & \text { Tomate Concombre } \\ & (1991)\end{aligned}$

$\begin{array}{llll}\text { BR 105 } & 90,0 \mathrm{a}(+31) & 81,3 \mathrm{~b}(+13) & 93,8 \mathrm{a}(+25) \\ \text { AGI } & 88,8 \mathrm{a}(+29) & 86,9 \mathrm{a}(+21) & 95,0 \mathrm{a}(+27) \\ \text { Tsp 2 } & 69,4 \mathrm{~b}(+1) & 80,0 \mathrm{~b}(+11) & 74,4 \mathrm{~b}(-1) \\ \text { Témoin } & 68,8 \mathrm{~b} & 71,9 \mathrm{c} & 75,0 \mathrm{~b}\end{array}$

à celle des plantes témoins ou des plantes élevées en présence de Tsp2 (tableau III). Enfin, le nombre de fleurs par plante après 2 mois de culture est significativement plus élevé chez la tomate et surtout chez le concombre (tableau IV); cependant, à ce stade, les masses sèches ne sont plus significativement différentes.

\section{Essai en conditions de production commerciale}

On observe très peu de mortalité dans les pensées repiquées chez le producteur horticole, quel

Tableau III. Effet en pots à la serre de l'incorporation de souches de Trichoderma dans le substrat de culture sur la masse de matière sèche (en $\mathrm{mg}$ ) produite 21 j après le semis par des tomates et des concombres (essais de 1991; moyennes de 16 répétitions). Dans chaque colonne, les masses suivies d'une même lettre ne sont pas différentes au seuil de $5 \%$, selon le test de Newman et Keuls.

\begin{tabular}{llllll} 
Traitements & \multicolumn{2}{c}{ Tomate } & & \multicolumn{2}{c}{ Concombre } \\
\cline { 2 - 3 } \cline { 5 - 6 } & $\begin{array}{c}\text { Parties } \\
\text { aériennes }\end{array}$ & Racines & & $\begin{array}{c}\text { Parties } \\
\text { aériennes }\end{array}$ & Racines \\
& & & & & \\
BR 105 & $390 \mathrm{a}$ & $110 \mathrm{a}$ & & $250 \mathrm{a}$ & $70 \mathrm{a}$ \\
AGl & $380 \mathrm{a}$ & $90 \mathrm{ab}$ & & $275 \mathrm{a}$ & $60 \mathrm{~b}$ \\
Tsp 2 & $240 \mathrm{ab}$ & $61 \mathrm{~b}$ & & $170 \mathrm{~b}$ & $30 \mathrm{c}$ \\
Témoin & $230 \mathrm{~b}$ & $60 \mathrm{~b}$ & $160 \mathrm{~b}$ & $30 \mathrm{c}$ \\
\hline
\end{tabular}

Tableau IV. Effet en pots à la serre de l'incorporation de souches de Trichoderma dans le substrat de culture sur le nombre moyen de fleurs par plante, dénombré 8 semaines après le semis des tomates ou des concombres (moyennes de 16 répétitions). Dans chaque colonne, les nombres suivis d'une même lettre ne sont pas différents au seuil de $5 \%$, selon le test de Newman et Keuls. Les chiffres entre parenthèses représentent les pourcentages de variation par rapport au témoin.

\begin{tabular}{llll}
\hline Traitements & $\begin{array}{c}\text { Tomate } \\
(1990)\end{array}$ & $\begin{array}{c}\text { Tomate } \\
(1991)\end{array}$ & Concombre \\
\hline BR 105 & $6,5 \mathrm{a}(+86)$ & $4,3 \mathrm{a}(+54)$ & $3,7 \mathrm{a}(+236)$ \\
AGI & $5,0 \mathrm{~b} \mathrm{(+43)}$ & $3,7 \mathrm{~b}(+32)$ & $3,1 \mathrm{~b}(+182)$ \\
Tsp 2 & $3,2 \mathrm{~cd}(-9)$ & $3,1 \mathrm{c}(+11)$ & $1,6 \mathrm{c}(+45)$ \\
Témoin & $3,5 \mathrm{c}$ & $2,8 \mathrm{~d}$ & $1,1 \mathrm{~d}$ \\
\hline
\end{tabular}

que soit le traitement : le taux de survie est compris entre 94 et $100 \%$. Le début de la floraison n'a pas été noté mais, lors du premier relevé, il y a 4 à 6 fleurs dans chacun des 3 lots traités, contre 2 dans le lot témoin. À la dernière notation, il n'y a que 12 fleurs chez les plantes témoins, chiffre significativement inférieur à ceux des lots traités. On dénombre respectivement 22,20 et 22 fleurs dans les lots traités par BR 105, par l'oxyquinoléine, et par BR 105 + l'oxyquinoléine. Cela représente des quantités moyennes de 0,35, 0,31 et 0,35 fleur par plante, respectivement, comparées à 0,2 chez les plantes non traitées.

\section{Antagonisme vis-à-vis de Pythium ultimum}

\section{Essais in vitro}

$\mathrm{Si}$, dans la presque totalité des cas, le rapport des surfaces occupées par le Trichoderma et par le Pythium est supérieur à 1, il apparaît néanmoins une grande diversité de comportement parmi les 113 souches de la collection. Dans $61,1 \%$ des confrontations, il y a interpénétration des thalles des 2 champignons. Chez $26,5 \%$ des couples, les 2 thalles s'arrêtent au contact l'un de l'autre. Enfin, dans $12,4 \%$ des cas, on observe un arrêt de la croissance alors que les thalles sont encore distants l'un de l'autre. Quatre souches seulement manifestent une forte action antagoniste se traduisant par une zone d'inhibition de plus de $1 \mathrm{~cm}$ : ce sont la 
souche de $T$ polysporum AGl et 3 des clones mutants issus de B 11 (dont BR 105). Pour 4 autres souches, la zone d'inhibition est de plusieurs millimètres.

Pour confirmer ces résultats, 4 des souches inhibitrices (BR 105, BR 107, AGl et KZ) et 5 souches non inhibitrices prises au hasard (MP, $A B W, A C C, O R N$ et $\mathrm{Jl}$ ) ont été à nouveau confrontées à $P$ ultimum. Les observations (tableau $V$ ) montrent la validité du classement précédent. AGI et BR 105 se montrent également, in vitro, fortement inhibitrices de $R$ solani et de $S$ rolfsii, alors qu'ABW ne manifeste pas d'action antagoniste; le clone ORN est faiblement inhibiteur de $S$ rolfsii (tableau $V$ ).

\section{Essais en serre}

Nous avons retenu pour ces essais les souches $A G I$ et BR 105, déjà remarquées précédemment pour leurs propriétés stimulatrices de croissance. La souche $A B W$, non inhibitrice in vitro, est utilisée comme témoin.

AGI et BR 105 assurent une bonne protection des concombres : leur effet favorable se traduit par la survie d'un plus grand nombre de plantes, mais aussi par un plus grand nombre de fleurs par plante au bout de 2 mois (tableau VI). Cependant, il n'apparaît pas de différences significatives dans la taille et le poids des plantes subsistantes. La souche ABW est totalement inefficace.

\section{Évolution des populations}

À la fin de l'essai précédent, après 8 semaines de culture, la densité d'inoculum de $P$ ultimum n'a pas varié par rapport à l'apport initial de 250 propagules $\mathrm{g}^{-1}$, en présence d'AGl et de BR 105 (tableau VII). Par contre, la souche ABW n'empêche pas la population pathogène de se multiplier et d'atteindre un niveau presque équivalent à celui du substrat non traité. Les populations de Trichoderma se maintiennent au niveau de l'inoculum introduit, sans différence nette entre les traitements.

On trouve des résultats analogues lorsque les champignons sont apportés dans un substrat non cultivé : dans ces conditions, la souche AGI réduit de près de $60 \%$ l'accroissement de la population de $P$ ultimum (fig 1 ). Les capacités infectieuses d'échantillons de substrat vis-à-vis des concombres varient en fonction du temps (fig 2), mais l'effet inhibiteur des souches AGI et BR 105 demeure très net pendant toute la durée de I'essai. La souche ABW n'a aucun effet inhibiteur.

\section{DISCUSSION}

II n'est pas difficile de trouver des souches de Trichoderma spp manifestant in vitro une activité antagoniste vis-à-vis de $P$ ultimum. Un tel mécanisme semble aussi bien fonctionner dans des

Tableau V. Antagonisme manifesté in vitro par des souches de Trichoderma vis-à-vis de 3 champignons parasites, après 36 h de confrontation. Le degré d'antagonisme est noté A3, A2 ou A1 (arrêt de croissance et zone d'inhibition respectivement supérieure ou égale à $10 \mathrm{~mm}$, comprise entre 5 et $10 \mathrm{~mm}$, ou inférieure à $5 \mathrm{~mm}$ ), $\mathrm{F}$ (cultures arrivant au contact) ou I (interpénétration des thalles). Le rapport des surfaces est calculé en divisant la surface du thalle de Trichoderma par celle du champignon parasite confronté.

\begin{tabular}{|c|c|c|c|c|c|c|c|c|c|}
\hline & $A G I$ & $B R 105$ & $B R 107$ & $K Z$ & $M P$ & $A B W$ & $A C C$ & ORN & $J$ \\
\hline \multicolumn{10}{|l|}{ Pythium ultimum } \\
\hline Degré d'antagonisme & A 3 & A 2 & A 2 & A 2 & $\mathrm{~F}$ & 1 & 1 & 1 & 1 \\
\hline Rapport des surfaces & 2,5 & 2,4 & 2,7 & 2,4 & 2,4 & 1,8 & 1,5 & 1 & 1 \\
\hline \multicolumn{10}{|l|}{ Rhizoctonia solani } \\
\hline Degré d'antagonisme & A 3 & A 3 & & & & I & & $\mathrm{F}$ & \\
\hline Rapport des surfaces & 2,5 & 1,3 & & & & 0,5 & & 0,2 & \\
\hline \multicolumn{10}{|l|}{ Sclerotium rolfsii } \\
\hline Degré d'antagonisme & A 3 & A 3 & & & & 1 & & A 1 & \\
\hline Rapport des surfaces & 2,5 & 1,3 & & & & 0,8 & & 1,1 & \\
\hline
\end{tabular}


Tableau VI. Effet sur des concombres de l'incorporation de souches de Trichoderma dans un sol contaminé par Pythium ultimum en pots à la serre. Les résultats suivis d'une même lettre ne sont pas différents au seuil de $5 \%$ selon le test de Newman et Keuls. Les chiffres entre parenthèses représentent les variations relatives (en \%) constatées par rapport aux plantes infectées par $P$ ultimum et non protégées.

\begin{tabular}{lll}
\hline Traitement & Taux de levée & $\begin{array}{c}\text { Nombre de } \\
\text { fleurs par plante }\end{array}$ \\
& & \\
\hline & & $1,1 \mathrm{a}$ \\
Pultimum & $18,4 \mathrm{~d}$ & $3,1 \mathrm{c}(+183)$ \\
Pultimum + BR 105 & $43,8 \mathrm{bc}(+138)$ & $2,5 \mathrm{~b}(+127)$ \\
Pultimum + AGl & $53,8 \mathrm{~b}(+192)$ & $1,4 \mathrm{ab}(+27)$ \\
Pultimum + ABW & $18,8 \mathrm{~cd}(+2)$ & $1,1 \mathrm{a}$ \\
Témoin non inoculé & $75,0 \mathrm{a}$ & \\
\hline
\end{tabular}

conditions naturelles. C'est ainsi que Lifshitz et al (1986) montrent que les processus de l'infection des semences de pois par des Pythium spp sont si rapides que seule une production de sub- stances inhibitrices dans la spermosphère peut expliquer l'effet protecteur assuré par un enrobage des graines avec des conidies de $T$ harzianum ou de $T$ koningii. Dans nos essais, les souches dont l'effet inhibiteur est le plus marqué in vitro sont aussi celles qui protègent le mieux les concombres de la fonte de semis. Par ailleurs, la présence simultanée dans un substrat organique de $P$ ultimum et de Trichoderma n'entraîne ni prolifération des Trichoderma, ni réduction de la population de Pythium à un niveau inférieur à son niveau initial, ce qui serait le cas s'il y avait mycoparasitisme.

Nos essais ont été réalisés seulement avec l'espèce $P$ ultimum, mais les résultats d'autres expérimentations montrent que l'antagonisme des Trichoderma vis-à-vis des Pythium n'est pas limité à une espèce particulière (Hadar et al, 1984). II serait cependant intéressant de vérifier que nos souches sont antagonistes non seulement des Pythium spp agents de fonte de semis, mais aussi des Pythium spp délétères qui, bien que faiblement parasites, contribuent

Tableau VII. Évolution de populations de Trichoderma et de Pythium ultimum, seules ou en mélange, sous une culture de concombres en pots, à la serre. Les Trichoderma et l'inoculum de $P$ ultimum sont incorporés dans le substrat respectivement 2 semaines et 1 semaine avant le semis des concombres. Les prélèvements pour le dénombrement des populations sont faits après 8 semaines de culture. Les chiffres entre parenthèses représentent les limites des intervalles de confiance à $5 \%$.

\begin{tabular}{|c|c|c|c|c|}
\hline \multirow[t]{2}{*}{ Traitements } & \multicolumn{2}{|c|}{$\begin{array}{l}\text { Inoculum en début de culture } \\
\text { (concentration théorique dans le sol) }\end{array}$} & \multicolumn{2}{|c|}{$\begin{array}{l}\text { Inoculum après } 8 \text { semaines de culture } \\
\text { (valeurs réellement mesurées) }\end{array}$} \\
\hline & P ultimum & Trichoderma & P ultimum & Trichoderma \\
\hline BR 105 & & $10^{8}$ & & $\begin{array}{c}2,8 \times 10^{8} \\
(2,1-3,6) \times 10^{8}\end{array}$ \\
\hline AGI & & $10^{8}$ & & $\begin{array}{c}5,0 \times 10^{7} \\
(2,9-7,1) \times 10^{7}\end{array}$ \\
\hline ABW & & $10^{8}$ & & $\begin{array}{c}2,8 \times 10^{8} \\
(2,1-3,6) \times 10^{8}\end{array}$ \\
\hline P ultimum & 250 & & $\begin{array}{c}1250 \\
(910-1590)\end{array}$ & \\
\hline P ultimum + BR 105 & 250 & $10^{8}$ & $\begin{array}{c}280 \\
(210-350)\end{array}$ & $\begin{array}{c}2,0 \times 10^{8} \\
(1,2-2,8) \times 10^{8}\end{array}$ \\
\hline$P$ ultimum + AGI & 250 & $10^{8}$ & $\begin{array}{c}230 \\
(205-255)\end{array}$ & $\begin{array}{c}4,5 \times 10^{7} \\
(3,1-5,9) \times 10^{7}\end{array}$ \\
\hline P ultimum + ABW & 250 & $10^{8}$ & $\begin{array}{c}1000 \\
(735-1265)\end{array}$ & $\begin{array}{c}4,5 \times 10^{7} \\
(3,1-5,9) \times 10^{7}\end{array}$ \\
\hline
\end{tabular}




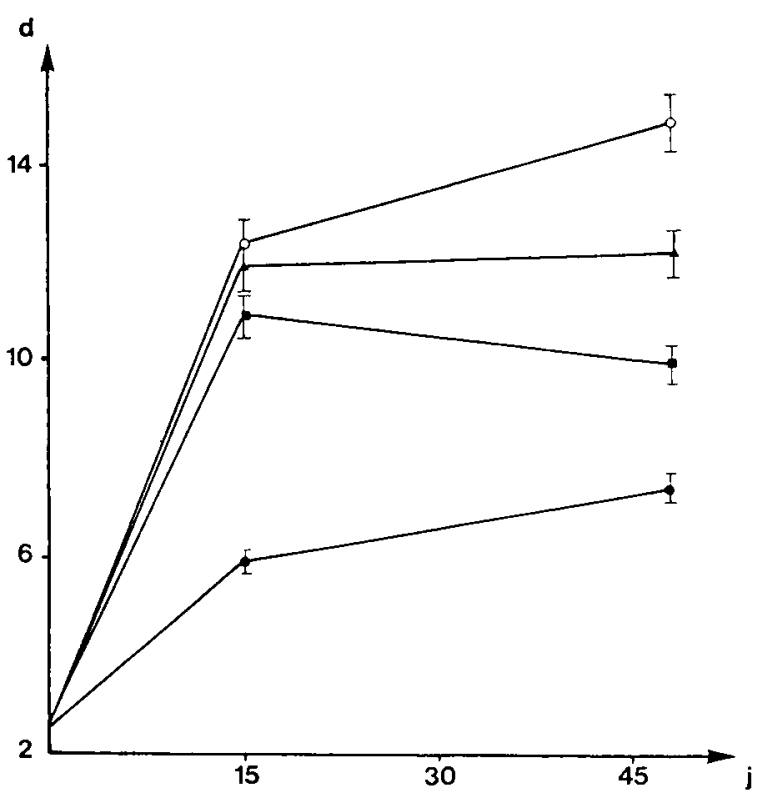

Fig 1. Effet de diverses souches de Trichoderma sur l'évolution de la densité d'inoculum (d) de Pythium ultimum dans un substrat organique (unités : $10^{2}$ propagules $\mathrm{g}^{-1}$ ). Traitements : $P$ ultimum $+T$ polysporum AGI (๑), $P$ ultimum $+T$ harzianum BR $105(\mathbb{D}), P$ ultimum + T harzianum ABW (A), $P$ ultimum seul (O). Les barres verticales représentent les écarts types de chaque série de mesures.

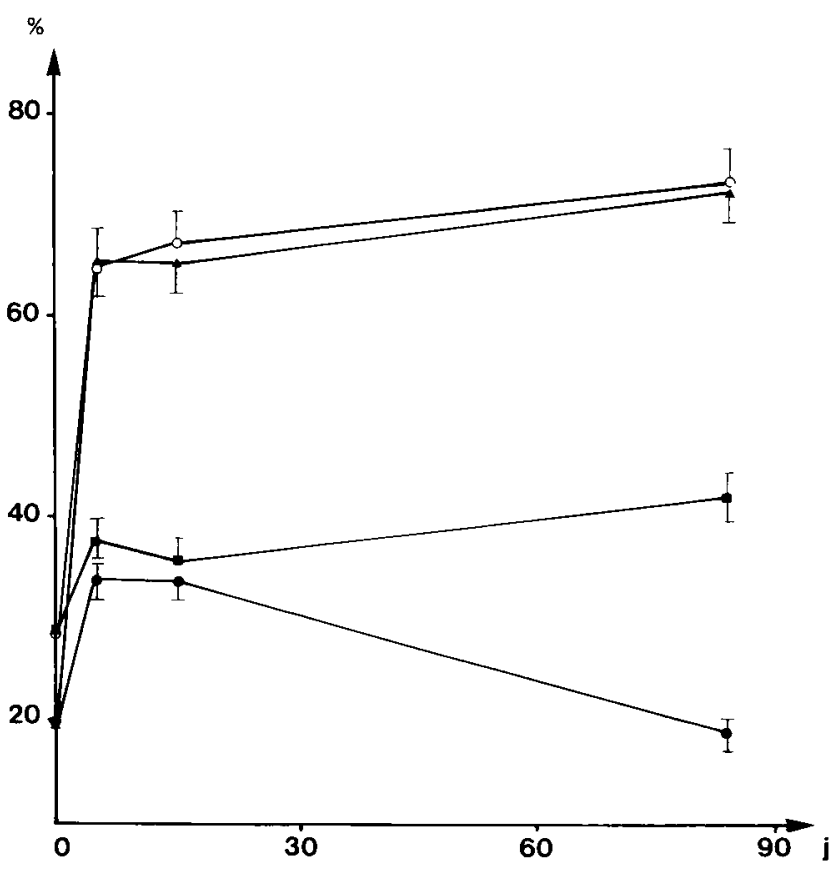

Fig 2. Effet de diverses souches de Trichoderma et du temps d'incubation dans le substrat sur la capacité infectieuse de Pythium ultimum. La capacité infectieuse est exprimée par le pourcentage de concombres tués après avoir été mis en contact, dans des conditions standard, avec des échantillons de substrat prélevés $0,5,15$ et 84 j après le début de l'essai. Traitements : $P$ ultimum $+T$ polysporum AGI $(\bullet), P$ ultimum $+T$ harzianum BR 105 (口), $P$ ultimum $+T$ harzianum ABW (A), $P$ ultimum seul (O). Les barres verticales représentent les écarts types de chaque série de mesures. par leur présence à un mauvais état général des plantes pendant toute la période de culture (Stanghellini et Kronland, 1986; Stanghellini et al, 1988). La forte inhibition que nous avons observée in vitro vis-à-vis de $R$ solani et de $S$ rolfsii peut être considérée comme un indice supplémentaire de la faible spécificité de l'effet fongistatique de nos isolats.

Il nous a été possible, d'autre part, de trouver dans notre collection des souches dotées de propriétés stimulantes. Cette stimulation se traduit par une meilleure germination in vitro et en serre, un développement accru et une floraison plus importante. Le développement concerne non seulement les parties aériennes, mais aussi les racines, et l'augmentation porte sur la masse de matière sèche, ce qui signifie qu'il ne s'agit pas simplement d'une absorption d'eau plus élevée, mais d'un accroissement général du métabolisme.

Nos résultats sont de même nature que ceux qui sont rapportés par Baker (1988), Lynch et al (1991) et Kleifeld et Chet (1992). Cependant, les effets stimulants signalés par ces auteurs n'ont été observés que chez des plantes cultivées dans de la terre ou des substrats de culture, mais pas en conditions axéniques contrôlées. Bien plus, le graphique présenté par Kleifeld et Chet (1992) montre que, en conditions stériles en boîtes de Petri, des graines de poivron ne germent pas mieux en présence de $T$ harzianum que des graines non traitées. Dans leurs essais, comme d'ailleurs dans ceux que nous présentons ici, le substrat de culture organique est préalablement stérilisé mais aucune précaution particulière n'est ensuite prise pour éviter sa recolonisation une fois que les pots sont disposés dans la serre. II est donc difficile d'affirmer que les effets observés dans ces conditions sont dus à une stimulation de croissance plutôt qu'à un effet protecteur du Trichoderma vis-à-vis de champignons délétères présents dans les substrats.

Aucun des auteurs précédents n'a utilisé comme référence un lot de plantes cultivées dans un substrat désinfecté avec un fongicide. Dans notre expérimentation avec des pensées (et dans d'autres essais non rapportés ici), en l'absence d'un traitement fongicide de référence, il aurait été possible de conclure que la plus grande précocité de floraison observée en présence de BR 105 était certainement due à un effet stimulant. Le fait qu'il y ait aussi une amélioration de la floraison dans les pots traités unique- 
ment à l'oxyquinoléine nous oblige à une grande prudence dans l'interprétation des résultats.

Cela ne signifie pas que nous excluons que certaines souches de Trichoderma spp puissent exercer un effet stimulant. Lindsey et Baker (1967), Windham et al (1986) l'ont mis en évidence dans des conditions gnotobiotiques; nous avons montré plus haut que les filtrats de culture stériles de plusieurs de nos isolats augmentaient le taux de germination et la masse de plantes semées sur un support gélosé autoclavé. Nous souhaitons simplement attirer l'attention sur le fait que l'interprétation de ces phénomènes complexes doit être faite avec précaution. Seules l'identification de composés responsables de la stimulation de croissance et la démonstration de leur effet sur des plantes cultivées en serre devraient permettre de conclure sur le mécanisme mis en jeu. Les travaux actuellement en cours permettent de penser qu'un tel mécanisme existe et qu'il est donc possible, comme nous nous le proposions, de trouver des isolats de Trichoderma à la fois stimulateurs de croissance et antagonistes de champignons pathogènes.

\section{REMERCIEMENTS}

Ce travail a été réalisé dans le cadre d'une bourse CIFRE (O Besnard) cofinancée par le MRT et la Société Letellier. Les auteurs remercient $\mathrm{J}$ Peyre pour la qualité de son assistance technique.

\section{RÉFÉRENCES}

Baker R (1988) Trichoderma spp as plant-growth stimulants. CRC Crit Rev Biotechnol 7, 97-106

Bouhot D, Ellal G (1976) Adaptation aux régions tempérées d'une technique de mesure de la densité d'inoculum de Pythium dans les sols. Ann Phytopathol 8, 241-243

Davet P (1979) Technique pour l'analyse des populations de Trichoderma et de Gliocladium virens dans le sol. Ann Phytopathol 11, 529-533

Gindrat D (1977) Effet de concentrations élevées de sels sur la croissance, la sporulation et la pigmenta- tion de Trichoderma spp. Can J Microbiol 23, 607616

Hadar Y, Harman GE, Taylor AG (1984) Evaluation of Trichoderma koningii and $T$ harzianum from New York soils for biological control of seed rot caused by Pythium spp. Phytopathology 74, 106-110

Harman GE, Taylor AG, Stasz TE (1989) Combining effective strains of Trichoderma harzianum and solid matrix priming to improve biological seed treatment. Plant Dis 73, 631-637

Kleifeld O, Chet I (1992) Trichoderma harzianum interaction with plants and effect on growth response. Plant Soil 144, 267-272

Lifshitz R, Windham MT, Baker R (1986) Mechanism of biological control of preemergence damping-off of pea by seed treatment with Trichoderma spp. Phytopathology 76, 720-725

Lindsey DL, Baker R (1967) Effect of certain fungi on dwarf tomatoes grown under gnotobiotic conditions. Phytopathology 57, 1262-1263

Lynch JM (1987) In vitro identification of Trichoderma harzianum as a potential antagonist of plant pathogens. Curr Microbiol 16, 49-53

Lynch JM, Wilson KL, Ousley MA, Whipps JM (1991) Response of lettuce to Trichoderma treatment. Lett Appl Microbiol 12, 59-61

Messiaen CM, Lafon R (1965) Les maladies des plantes maraichères. INRA, Paris, tome 2

Ricci P, Toribio JA, Messiaen CM (1976) La dynamique des populations de Pythium dans les sols maraîchers de Guadeloupe. I. Méthodes d'étude. Ann Phytopathol 8, 51-63

Sivan A, Elad Y, Chet I (1984) Biological control effects of a new isolate of Trichoderma harzianum on Pythium aphanidermatum. Phytopathology 74, 498-501

Stanghellini ME, Kronland WC (1986) Yield loss in hydroponically grown lettuce attributed to subclinical infection of feeder rootlets by Pythium dissotocum. Plant Dis 70, 1053-1056

Stanghellini ME, White JG, Tomlinson JA, Clay C (1988) Root rot of hydroponically grown cucumbers caused by zoospore - producing isolates of $\mathrm{Py}$ thium intermedium. Plant Dis 72, 358-359

Whipps JM, Lumsden RD (1991) Biological contral of Pythium species. Biocont Sci Technol 1, 75-90

Windham MT, Elad Y, Baker R (1986) A mechanism for increased plant growth induced by Trichoderma spp. Phytopathology 76, 518-521 